\title{
Risk of spinal haematoma caused by epidural catheters after major upper gastrointestinal surgery: normal postoperative changes in coagulation, and the relationship between advanced and routine tests of coagulation.
}

\author{
Owain Thomas MD MA(Cantab) PhD ${ }^{1,2}$, Hampus Rein MD ${ }^{3,4}$ Emanuel Lybeck MD ${ }^{4,5}$ \\ Karin Strandberg MD PhD ${ }^{6}$, Ulf Schött MD PhD ${ }^{2,7}$
}

Contact: odt@cantab.net. 1Dept of Paediatric Anaesthesiology \& Intensive Care, SUS Lund University Hospital, Sweden 2 Dept of Anaesthesiology and Intensive Care, Institute of Clinical Sciences, Lund, University of
Lund, Sweden, 3 Swedish Foreign Department, Brussels 4 Previously medical student, Faculty of Medicine, University of Lund, Sweden 5 Kalmar County Health Authority, Sweden. 6 Coagulation Laboratory, Dept of
Clinical Chemistry, Division of Laboratory Medicine, Skåne University Hospital, Malmö, Sweden 7 Department of Anaesthesia and Intensive Care, SUS Lund University Hospial, Sweden. Background

Routine coagulation tests are often taken to estimate the risk of spinal haematoma upon withdrawal of epidural catheters. Previous research shows that the first few days after operation are characterised by apparent mild hypocoagulation as shown by the routine tests PT-INR (prothrombin international normalised ratio) and aPTT (activated partial thromboplastin time) while postoperative patients are otherwise known to be prone to thrombosis. Guidelines are inconsistent on what to do when test results indicate slight hypocoagulation yet there is a clinical indication to remove the catheter.

We conducted routine and advanced tests, hypothesizing that patients undergoing major upper gastrointestinal (GI) surgery would be deficient in vitamin K-dependent coagulation factors, or hypocoagulative because of accumulation of low molecular weight heparin (LMWH).

\section{Methods}

27 men and 11 women scheduled for epidural analgesia for major upper GI surgery were included. Blood tests
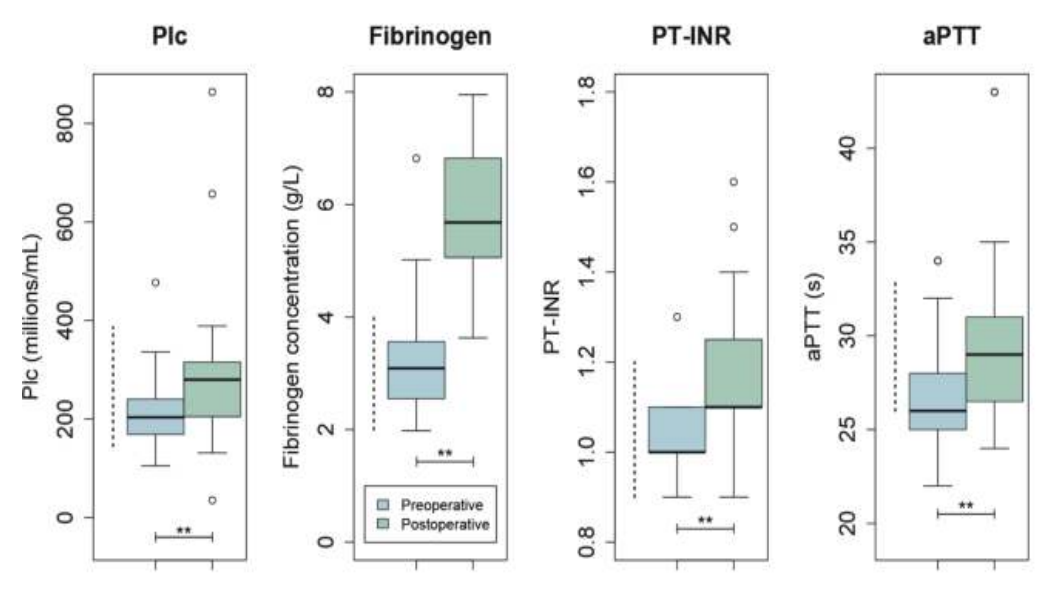
were taken from each patient on two occasions: prior to epidural catheterization and at the time of epidural catheter withdrawal.

The following assays were run on each occasion:

Routine tests: PT-INR, aPTT and platelet count (PIc), albumin. Advanced tests: proteins induced by vitamin $\mathrm{K}$ absence (PIVKA), rotational thromboelastometry $\left(\right.$ ROTEM $^{\circ}$ EXTEM, INTEM FIBTEM and HEPTEM), multiple electrode aggregometry (Multiplate ${ }^{\circ}$ ) and activites of factor II, VII, IX, X, XI, XII and XIII.

Pre- to postoperative results were compared using Wilcoxon's signed ranked test and correlations were tested using Pearson's (parametric) product-moment and Spearman's (nonparametric) rank correlation tests.

\section{$\underline{\text { Results and discussion }}$}

Catheters were withdrawn after a mean of $6.2 \pm 3.2$ days, when all patients had elevated CRP indicating postoperative inflammation. The mean duration of operation was 10.4 hours and the mean blood loss during operation was $400 \mathrm{ml}$ (range $50-4650 \mathrm{ml}$ ). 5 patients gave preoperative but not postoperative samples.

References Thomas et al. Coagulative safety of epidural catheters after major upper gastrointestinal surgery: advanced and routine coagulation analysis in 38 patients Perioperative Medicine (2016) 5:28. Breivik et al. Nordic guidelines for neuraxial blocks in disturbed haemostasis from the Scandinavian Society of Anaesthesiology and Intensive Care Medicine. Acta Anaesthesiol Scand. $2010 ; 54: 16-41$.

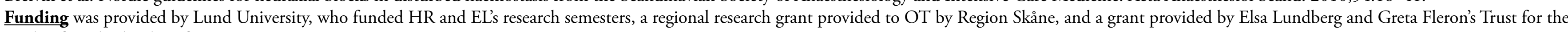
Study of Medical Side-Effects. renal failure, major blood loss or dilution, and anticoagulant
Postoperative thrombocytosis and hyperfibrinogenaemia were demonstrated, as were slight but significant increases in PT-INR and aPTT (see diagrams). All mean ROTEM$\mathrm{MCFs}$ (maximal clot firmnesses) increased significantly above their reference ranges increasing hypercoaglability, especially FIBTEM which reflects fibrinogen levels. All mean pre- and postoperative ROTEM clotting times were within their reference ranges, such that the clinical relevance of thromboelastometry in the context of this article remains unclear.
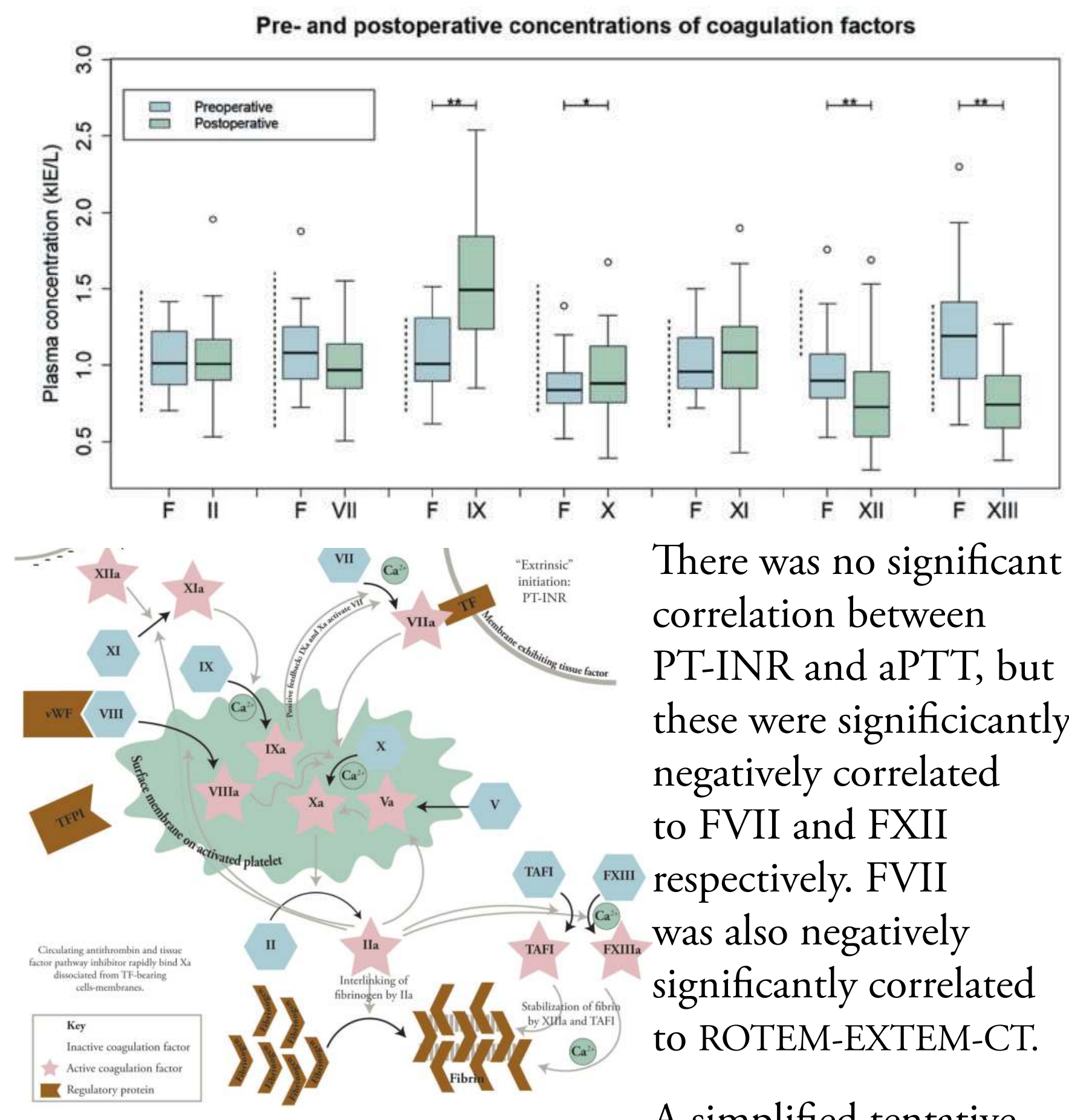

There was no significant correlation between PT-INR and aPTT, but these were significicantly negatively correlated to FVII and FXII respectively. FVII was also negatively significantly correlated to ROTEM-EXTEM-CT.

A simplified tentative conclusion is that postoperative aPTT is prolonged by low postoperative FXII and postoperative PT-INR by low FVII. Given that complete congenital deficiency of FXII results in prolonged aPTT but not clinical coagulopathy, epidural catheters may likely be withdrawn despite slightly prolonged aPTT. A condition for this must be the absence of other risk factors for coagulopathy such as preexisting haemophilia, drugs. Prolonged PT-INR may be more clinically significant since complete absence of FVII does cause coagulopathy.

The decrease in FXIII that we observed cannot be detected by aPTT or PT-INR - some patients had clinically low postoperative levels of FXIII. The postoperative hyperfibrinogenaemia observed in this study may compensate for low FVII and FXIII. 